\title{
Electron Microscopy of the Microtubule Framework in Primary Cilia
}

\author{
Shufeng Sun ${ }^{1,2}$, Rebecca L. Fisher ${ }^{1}$, Brian T. Pentecost ${ }^{1}$ and Haixin Sui ${ }^{1,2^{*}}$ \\ 1. Wadsworth Center, New York State Department of Health, Albany, NY, USA. \\ 2. Department of Biomedical Sciences, School of Public Health, University at Albany, Albany, NY, \\ USA. \\ * Corresponding author: haixin.sui@health.ny.gov
}

Primary cilia of kidney cells are microtubule-based sensory organelles extending into extracellular space. They detect environmental signals and influence physiological activities of the cells. Defects of primary cilium assembly or dysfunctions of sensory proteins of primary cilia are linked to many developmental defects and clinical disorders (ciliopathies) [1]. Accurate structural information of primary cilia is fundamental for understanding the assembly, maintenance and sensory functions of primary cilia. There are only a handful reports with conventional electron microscopic (EM) data about primary cilium structure. In contrast, structural data using both conventional and cryo-EM is abundant for motile cilia. Therefore, much of our structural understanding of primary cilia is based upon, or borrowed from, the knowledge obtained with motile cilia. The frameworks (axoneme) of both motile and primary cilia are microtubule-based complexes (MtCs). Microtubules present in singlet [2-5], doublet [6-8], and triplet [9] forms in the axoneme. The 3D structure of primary cilium axoneme has been described as a cylindrical array of nine microtubule doublets (the $9+0$ paradigm), which is the same as or similar to the cylindrical array of the 9 peripheral doublets in motile cilia. The $9+0$ paradigm is currently the accepted structural baseline of primary cilium research. However, there are several EM reports where the data conflict with the $9+0$ paradigm. Lack of direct 3D structural data of primary cilia is due to technical challenges in EM specimen preparation.

We have successfully developed novel approaches to overcome the technical challenges of EM specimen preparation of primary cilia in kidney cells. This allowed us to study epithelial primary cilia using methods of electron tomography. In our study, cryo-electron tomography of primary cilia was accomplished after a technical breakthrough in preparing vitreously frozen primary cilium specimens. We prepared cryo-EM grids by culturing kidney cells on EM grids. The grids were plunge-frozen after cells produced primary cilia. To our surprise, our cryo-electron tomographic (cryo-ET) data was not compatible with the $9+0$ paradigm. In order to obtain the 3D structure of the entire length of primary cilia, we employed the method of serial section electron tomography (SSET)[10]. SSET works with plastic serial sections. We developed a method to protect primary cilia from breaking off during the substitution and plastic embedding processes of specimen preparation. SSET generally combines multiple electron tomograms of serial sections to create 3D structural maps for large subcellular assemblies that are difficult or impossible to fit in sections of a thickness that electron beam can penetrate. These methodological advances enabled us to successfully obtain 3D structural maps for a large number of primary cilia. Our work provides the first complete 3D structural model of typical epithelial primary cilia as an accurate structural baseline for primary cilium research and offers rich new insights into many fundamental questions about primary cilium biology [11]. 
References:

[1] F Hildebrandt, T Benzing and N Katsanis, New England Journal of Medicine 364 (2011), p. 1533.

[2] E Nogales et al., Cell 96 (1999), p. 79.

[3] H Li et al., Structure 10 (2002), p. 1317.

[4] H Sui and KH Downing, Structure 18 (2010), p. 1022.

[5] GM Alushin et al., Cell 157 (2014), p. 1117.

[6] H Sui and KH Downing, Nature 442 (2006), p. 475.

[7] D Nicastro et al., Proc Natl Acad Sci U S A 108 (2011), p. E845.

[8] M Ichikawa et al., Nat Commun 8 (2017), p. 15035.

[9] S Li et al., EMBO J 31 (2012), p. 552.

[10] GE Soto et al., NeuroImage 1 (1994), p. 230.

[11] We thank Dr. Jie He and Ms. Chyongere Hsieh for their assistance in the early stage of cryo-EM testing. This work is supported by the National Institutes of Health GM101026 to HS.
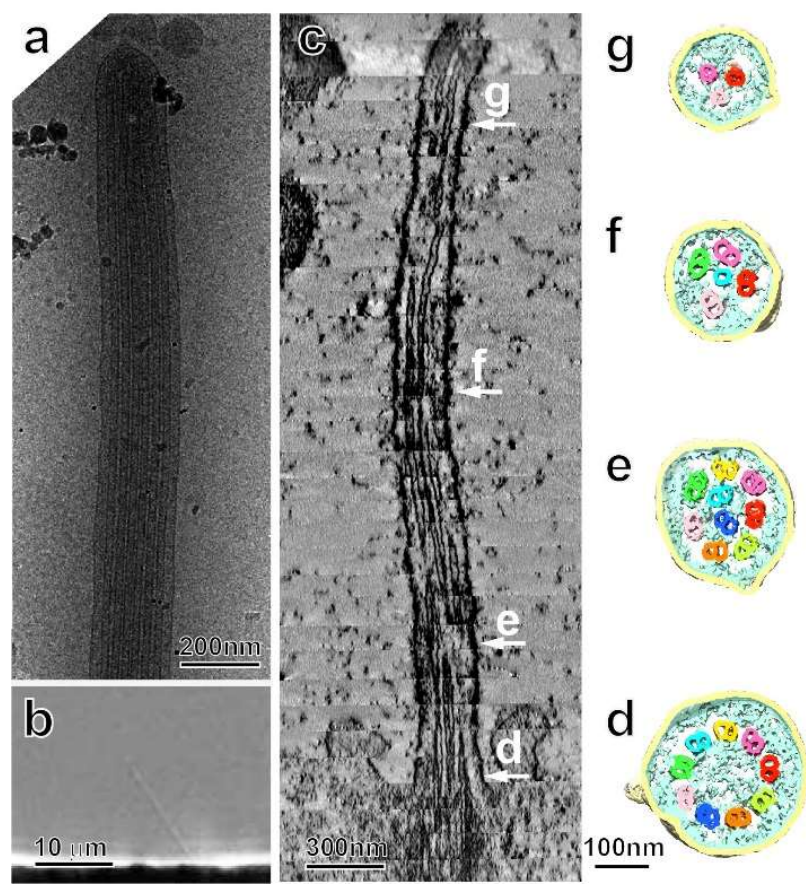

Figure 1. Electron microscopy of primary cilia: (a) EM micrography of a vitreously frozen primary cilium. (b) A primary cilium, at the edge of an EM grid bar, observed by differential interference contrast (DIC) imaging. (c) A longitudinal section of a primary cilium 3D map, for which the cross sections are shown in (d), (e), (f), and (g), respectively. 